\title{
Aplicación del Mapa de Preferencia Externo en la Formulación de una Bebida Saborizada de Lactosuero y Pulpa de Maracuyá
}

\author{
Carlos García-Mogollon ${ }^{(1)^{*}}$, Armando Alvis-Bermudez ${ }^{(2)}$ y Pedro Romero ${ }^{(2)}$ \\ (1) Universidad de Sucre, Facultad de Ingeniería, Sincelejo, Sucre-Colombia. \\ (e-mail: carlos.garcia@unisucre.edu.co) \\ (2) Universidad de Córdoba, Programa de Ingeniería de Alimentos, Facultad de Ingenierías, Grupo de \\ Investigación Procesos y Agroindustria de vegetales, Carrera 6 № 76-103, Km 3, vía Cereté. Córdoba, \\ Colombia.
}

${ }^{*}$ Autor a quien debe ser dirigida la correspondencia.

Recibido Abr. 22, 2015; Aceptado Jul. 2, 2015; Versión final Jul. 6, 2015, Publicado Oct. 2015

\begin{abstract}
Resumen
Se elaboraron bebidas refrescantes a base de lactosuero variando las concentraciones de pulpa de maracuyá de $8.0,11.5$ y $15 \%$ y azúcar de 5.0, 7.5 y 10\%. La evaluación instrumental consistió en la determinación de $\mathrm{pH}$, acidez y ${ }^{\circ}$ Brix. El estudio de consumidores se efectuó con 60 personas aplicando una escala hedónica de 5 puntos. La correlación de los datos instrumentales-sensoriales-hedónicos se efectuó mediante un mapa de preferencias externo. En las bebidas el pH fue superior a 4.0 y la acidez mayor a $0.2 \%$. Se establecieron tres segmentos de consumidores y para las formulaciones con pulpa al $8 \%$ con $5 \%$ y $7.5 \%$ de azúcar, el consumidor las identificó por su pH; la 11.5\%:10\% (pulpa:azúcar) la asoció por los ${ }^{\circ}$ Brix, las 15\%:7.5\% y 11.5\%:7.5\% las asoció con la acidez. Las formulaciones 15\%:7.5\%, 15\%:10\% y $11.5 \%: 10 \%$ fueron preferidas por los consumidores, lo que se relaciona con un sabor más dulce e intenso de esta formulación.
\end{abstract}

Palabras clave: lactosuero; bebidas refrescantes; passiflora edulis; análisis sensorial

\section{Application of the External Preferences Map in the Formulation of a Flavored Drink made from Whey and Passion Fruit Pulp}

\begin{abstract}
Refreshing drinks from whey were developed, varying the concentrations of pulp of passion fruit of 8.0, 11.5 and $15 \%$ and sugar $5.0,7.5$, and $10 \%$. The Instrumental evaluation consisted of a determination of $\mathrm{pH}$, acidity and ${ }^{\circ}$ Brix. The consumer study was carried out with 60 people by applying a five-point hedonic scale. The instrumental-sensory-hedonic relationship is established through the external preferences map. In all drinks $\mathrm{pH}$ was greater than 4.0 and acidity greater than $0.2 \%$. Three consumer segments were established and for the formulations with pulp at $8 \%$ and $5 \%$ and $7.5 \%$ sugar, consumers identify them by their $\mathrm{pH}$; the $11.5 \%: 10 \%$ (pulp:sugar) were associated by the ${ }^{\circ} \mathrm{Brix}$, the $15 \%: 7.5 \%$ and $11.5 \%: 7.5 \%$ were associated by the acidity. Formulations 15\%:7.5\%, 15\%:10\% and 11.5\%:10\% were preferred by consumers, fact that is related to the sweeter and more intense flavor of this formulation.
\end{abstract}

Keywords: whey; refreshing drinks; passiflora edulis; sensory analysis 


\section{INTRODUCCIÓN}

El lactosuero es un líquido de color amarillo verdoso derivado de la coagulación de la caseína de leche fresca mediante tratamiento enzimático para obtener queso. El lactosuero está compuesto de materia orgánica constituida por $4,8 \%$ de lactosa, $0,75 \%$ de proteínas y $6,2 \%$ de materia seca. Se ha constituido en el principal residuo de la industria láctea, donde una parte de éste es usado para alimentación animal y el resto tratado como un desecho vertido directamente en los cursos de agua naturales (García et al., 2013). Por tanto, existe la necesidad de eliminar un vertido altamente contaminante a través de la conversión a productos de valor agregado que permitan superar el problema ambiental y generar una alternativa alimentaria (Hernández y Matos, 2011) para propósitos nutricionales (Conti et al., 2012) y terapéuticos que se traduzcan en beneficios económicos para la industria láctea (Pandiyan et al., 2011; Acevedo et al., 2014). El mercado de las bebidas está en crecimiento a nivel mundial y el lactosuero ha sido reconocido como una materia prima en la producción de bebidas refrescantes, saludable y nutritiva. Conscientes de esto recomiendan evaluar las características sensoriales y estimar el potencial de aceptación de una bebida refrescante de naturaleza isotónica a partir de lactosuero.

La fabricación de bebidas a base de suero de leche requiere la mezcla de jugo de frutas apropiadas y mínimamente procesadas con selección de estabilizadores adecuados y acidulantes para elaborar bebidas de fruta aceptables basadas en suero. Krasaekoopt y Lokuliyanage (2011) usaron lactosuero de leche de vaca, búfalo y de cabra para elaborar una bebida fermentada, Sakhale et al. (2012) utilizaron una bebida saborizada con mango, Singh y Singh (2012) obtuvieron una bebida energética con pulpa de mango, Shukla et al. (2013) probióticos y jugo de piña, Sameen et al. (2013) bebida carbonatada saborizada. La maracuyá (Passiflora edilus) fruto de la flor de la pasión es originario del Brasil, es deliciosa, exótica y nutricionalmente aporta vitamina C $12 \mathrm{mg} / 100 \mathrm{~g}$ y el jugo constituye el $36,8 \%$ de la fruta, presenta una acidez titulable del 44,7\% como ácido cítrico, pH entre 2,8 - 3,3 y ${ }^{\circ}$ Brix de 12-14 (ICONTEC NTC 404). El lactosuero es un subproducto que se genera en grandes cantidades y contiene alrededor del $55 \%$ del total de nutrientes de la leche. Sin embargo, este líquido está subutilizado debido al poco conocimiento sobre sus usos potenciales, por tanto es desechado como efluente lo que genera un grave impacto ambiental debido a su alta demanda biológica de oxígeno o se utiliza como alimento para porcinos, lo cual no genera muchas utilidades para las queserías.

Basado en las cualidades nutricionales del lactosuero y de la pulpa de maracuyá este estudio tiene como objetivo la formulación de una bebida saborizada incorporando azúcar (5, 7,5 y 10\%) y pulpa de maracuyá $(8,0,11,5$ y $15,0 \%)$ y evaluar sus propiedades fisicoquímicas, la preferencia de consumidores y correlacionarlas mediante un Mapa de Preferencia Externo.

\section{MATERIALES Y MÉTODOS}

\section{Formulación}

Para la elaboración de la bebida se tomó una base 100 de lactosuero dulce desproteinizado $(\mathrm{pH} 6,6)$ para formular la pulpa de maracuyá con 3,48\% ácido cítrico, $12,4{ }^{\circ}$ Brix y el azúcar (Tabla 1). Para considerarla refresco de fruta, la pulpa debe estar en proporción mínima de $8 \%$ masa/masa, acorde al capítulo V: artículo 27 de la Resolución 7992 de 1991. Los aditivos como benzoato de sodio en dosis máxima de 100 ppm y CMC estabilizante máximo $2 \mathrm{~g} / \mathrm{l}$.

Tabla 1: Composición (\%) de ingredientes en las formulaciones.

\begin{tabular}{|c|c|}
\hline Azúcar & Pulpa \\
\hline $\mathrm{X} 1$ & $\mathrm{X} 2$ \\
\hline 5,0 & 8,0 \\
\hline 7,5 & 11,5 \\
\hline 10 & 15,0 \\
\hline
\end{tabular}

\section{Proceso de elaboración}

El proceso de elaboración de la bebida refrescante se procedió acorde al procedimiento propuesto Londoño et al. (2008) en la cual se toman las muestras del lactosuero fresco para las mediciones de pH y acidez, se procede a hacer una filtración con una malla filtro, se aplica un calentamiento rápido a $90{ }^{\circ} \mathrm{C}$ durante 10 minutos de modo que se produzca una desnaturalización de la $\beta$-lactoglobulina, proteína sérica termolábil 
sospechosa de posibles reacciones alérgicas en humanos (Choéz, 2010). Se enfría hasta $60^{\circ} \mathrm{C}$ y se filtra. Cuando el lactosuero estaba a una temperatura de $50{ }^{\circ} \mathrm{C}$ se adicionaron la CMC y azúcar (previamente mezclados), luego se adicionó benzoato de sodio y la mezcla se agitó. A la mezcla se le realizó un calentamiento a $70{ }^{\circ} \mathrm{C}$ durante $5 \mathrm{~min}$, seguido de un enfriamiento hasta $41^{\circ} \mathrm{C}$, luego se adicionó la pulpa y se envasó. La bebida obtenida se almacenó en refrigeración a una temperatura de $4 \pm 1{ }^{\circ} \mathrm{C}$. Para la caracterización fisicoquímica de las materias primas y la bebida se determinaron los sólidos solubles ( $\left.{ }^{\circ} B r i x\right)$ utilizando un Refractómetro Mettler Toledo 30PX (AOAC, 954.10); el pH se midió con un potenciómetro digital marca Mettler Toledo Five-Easy según el método oficial (AOAC 954.10); la acidez se determinó por titulación con $\mathrm{NaOH}(0,1 \mathrm{~N})$ expresándose como porcentaje de ácido cítrico (AOAC 954.10).

\section{Evaluación sensorial}

Las formulaciones de la bebida refrescante fueron evaluadas mediante el grado de satisfacción de consumidores basándose en las características de olor, color, sabor y aceptación general, utilizando una escala hedónica de 5 puntos con los siguientes descriptores: Me desagrada mucho=1, Me desagrada un poco $=2$, Ni me gusta ni me disgusta $=3$, Me gusta $=4$ y Me gusta mucho $=5$. Las muestras se presentaron con un volumen de $10 \mathrm{ml}$, con un panel de 60 evaluadores consumidores. Las muestras fueron servidas a los consumidores en vasos de 1.5 onzas a una temperatura menor a $4{ }^{\circ} \mathrm{C}$. Se realizaron la agrupación de consumidores mediante la Clasificación Jerárquica Ascendente (CJA) para la formación de clases o segmentos. Se usó el Análisis de Componentes Principales (ACP) para la correlación de los datos instrumentales-sensoriales para la construcción del Mapa de Preferencias Externo (MEP) modelo vectorial y circular en asociación con el coeficiente de determinación $\left(R^{2}\right)$ para la búsqueda de características sensoriales y puntos ideales que produzcan un incremento o decremento del estímulo en el espacio de la preferencia.

\section{Diseño experimental}

Se aplicó un diseño completamente al azar (ANOVA) con arreglo factorial $2^{3}$ para las variables pulpa de maracuyá de 3 niveles (8, 11.5 y 15\%) y azúcar con 3 niveles $(5,7.5$ y 10\%). Se realizaron 3 repeticiones para un total de 27 unidades experimentales para los resultados de la caracterización físico-química de la bebida y el test de Tukey al nivel de significancia del $5 \%$ si existen diferencias entre las formulaciones. Los datos fueron analizados por el programa Statgraphics Centurion XVI.

\section{RESULTADOS Y DISCUSIÓN}

\section{Caracterización de materias primas}

Las propiedades fisicoquímicas del lactosuero de esta investigación y otros autores se muestran en la tabla 2 , en ella se observa que el $\mathrm{pH}$, la acidez y los ${ }^{\circ}$ Brix obedecen a un lactosuero fresco. Se destaca el pH bajo usado por Pandiyan et al. (2011) y Sammen et al. (2013) correspondiente a lactosuero acidificado o no fresco con una alta acidez. Los sólidos solubles no determinan el tipo de lactosuero; sin embargo, se muestra que se usa lactosuero fresco para elaborar bebidas y acidificado para elaborar bebidas fermentadas.

Tabla 2: Propiedades fisicoquímicas del lactosuero fresco por varios autores

\begin{tabular}{|l|c|c|c|}
\hline \multicolumn{1}{|c|}{ Autor } & $\mathrm{pH}$ & Acidez (\% de ácido láctico) & ${ }^{\circ}$ Brix \\
\hline Esta investigación & $6.59 \pm 0.05$ & $0.12 \pm 0.00$ & $6.71 \pm 0.1$ \\
\hline Choez, 2010 & 6.6 & - & - \\
\hline Alvarez, 2013 & 6.29 & $0.12-0.39$ & - \\
\hline Pandiyan et al., 2011 & 5.0 & - & - \\
\hline Mazhuvanchery y Kulkarni, 2013 & 6.2 & 0.13 & - \\
\hline Shukla et al., 2013 & - & 0.18 & - \\
\hline Sammen et al., 2013 & 4.6 & 0.5 & 7.66 \\
\hline
\end{tabular}

La pulpa de maracuyá utilizada tiene $\mathrm{pH}$ de $3,10 \pm 0,054$, acidez $3,48 \pm 0,069 \%$ como ácido cítrico y sólidos solubles $12,43 \pm 0,15^{\circ}$ Brix. Los datos obtenidos se encuentran ligeramente por debajo de los datos reportados por Cerquera et al. (2012), para lo cual el pH es de $2.9 \pm 0.15$, los sólidos solubles (ํㅏix) $15.0 \pm 1.78$ y la acidez total titulable $4.42 \pm 0.89 \%$ como ácido cítrico. 
El análisis de varianza para la variable acidez con respecto a los porcentajes de pulpa y azúcar mostraron diferencias estadísticamente significativas $(p<0.05)$, en el $\mathrm{pH}$ solo el factor azúcar $(p<0.05)$ y en los sólidos solubles ( ${ }^{\circ}$ Brix) el factor pulpa $(p<0.05)$. La interacción de los factores no tuvo efecto en las variables. Las pruebas de múltiples rangos para $\mathrm{pH}$ determinan que las medias de cada nivel para este factor difieren entre sí, observando que para cada formulación a medida que aumenta el porcentaje de azúcar disminuye la media del $\mathrm{pH}$ de la bebida, condición que se atribuye a las propiedades de la pulpa de maracuyá que presentó un $\mathrm{pH}$ bajo $(3.1 \pm 0.05)$. El rango de $\mathrm{pH}$ en las formulaciones va de $4.21(15 \%: 7.5 \%)$ a $4.06(11.5 \%: 10 \%)$ y según la resolución 3929 de 2013 el pH para un refresco de fruta debe ser máximo 4.0, siendo las formulaciones 8\%:10\% y $11.5 \%: 10 \%$ las más cercanas a esta especificación.

La prueba de múltiples rangos para determinar las diferencias entre las medias de acidez por el porcentaje de azúcar mostró tres grupos homogéneos y para la pulpa dos grupos homogéneos (grupo con 15\% pulpa y el grupo con $11.5 \%$ y $8.0 \%$ pulpa). Según la resolución 3929 de 2013 la acidez titulable expresada como ácido cítrico, para refrescos de frutas, debe ser mínimo de $0.2 \%$, así tenemos que todas las formulaciones cumplen con esta especificación, presentándose el menor porcentaje con una acidez de $0.28 \pm 0.012 \%$ en la formulación con pulpa al $8 \%$ y $5 \%$ de azúcar. Coto y Flóres (2011) reportaron una acidez de $0.3 \%$ ácido cítrico para una bebida saborizada con maracuyá, al compararlo con los obtenidos se observa que en la mayoría de las formulaciones la acidez sobrepasa este valor debido al porcentaje de pulpa utilizado. Aunque el efecto del porcentaje de azúcar sea menos influyente que el de pulpa, ya que se observa que la acidez de la bebida presenta mayor variación cuando el porcentaje de pulpa varía. Esto se debe principalmente a la naturaleza ácida de la pulpa de maracuyá (3,48\% como ácido cítrico).

Los sólidos solubles en la bebida aumentan con el aumento de la concentración de pulpa, siendo de $11.51 \pm 0.1^{\circ}$ Brix, $13.47 \pm 0.1^{\circ}$ Brix y $15.3 \pm 0.1^{\circ}$ Brix para $8 \%, 11.5 \%$ y $15 \%$ de pulpa respectivamente. La bebida 15\%:10\% si comparamos estos datos con las especificaciones de la Resolución 3929 de 2013 tenemos que las formulaciones con $11.5 \%$ y $15 \%$ de pulpa no cumplen con la especificación que debe ser como máximo $13{ }^{\circ}$ Brix. Los sólidos de las formulaciones en cuestión (8\%:10\%, 11.5\%:10\% y 15\%:10\%) difieren de los reportados por Lagua (2011) cuyos datos para sólidos solubles en sus diferentes formulaciones oscilan entre 17.8 y $19.91^{\circ}$ Brix. Al comparar con los datos reportados por Pilaquinga (2012) los cuales oscilan en un rango entre 14.1 y $14.4^{\circ}$ Brix para las bebidas con diferentes pulpas, vemos que las formulaciones que más se acercan son $8 \%: 7.5 \%, 11.5 \%: 7.5 \%$ y el $15 \%: 7.5 \%$ las cuales poseen un porcentaje de azúcar del $7.5 \%$, porcentaje que varía un poco del utilizado por Pilaquinga, (2012) el cual utiliza porcentajes entre el $3 \%$ y el $7 \%$. Pandiyan et al. (2011) elaboraron una bebida de lactosuero saborizada con pulpa de mango al $4 \%, 5 \%$ y $6 \%$ con sólidos de 5,75 a $5,92 \%$.

\section{Mapa de preferencia}

El ACP (Análisis de Componentes Principales) muestra en el círculo de correlación (Figura 1) que los dos ejes principales se encontraron con el $99.81 \%$ de la variación total de los datos, observando que las formulaciones con un $8 \%$ y $15 \%$ de pulpa contribuyeron en la formación de $\mathrm{F} 1$; mientras las formulaciones con $11.5 \%$ de pulpa lo hacen para F2. Los atributos físico-químicos como pH y acidez contribuyen en una proporción homogénea con $\mathrm{F} 1$ y los ${ }^{\circ}$ Brix contribuyen principalmente con F2. La variable acidez se correlaciona negativamente con el $\mathrm{pH}$ y los ${ }^{\circ} \mathrm{Brix}(-0.992$ y $-0.074 \mathrm{p}<0.05)$, mientras el $\mathrm{pH}$ se correlacionó positivamente con los ${ }^{\circ}$ Brix $(0.013, p<0.05)$. Las formulaciones con pulpa al $8 \%$ con $5 \%$ y $7.5 \%$ de azúcar el consumidor

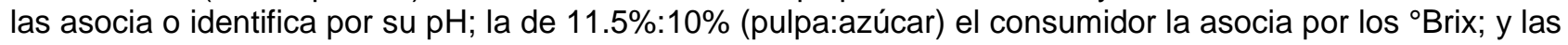
formulaciones $15 \%: 7.5 \%, 11.5 \%: 7.5 \%$ se asocian con la acidez.

El análisis de ANOVA estableció diferencias significativas $(p<0.05)$ de las formulaciones respecto a las respuestas hedónicas en la aceptación general. La relación entre evaluaciones sensoriales por consumidores y los datos instrumentales dan información de las diferencias sobre las preferencias de los consumidores (Gámbaro et al., 2007). Se identificaron 3 segmentos de consumidores (Figura 2) mediante la aplicación de la Clasificación Ascendente Jerárquica para los datos de aceptación general. El segmento 1 se conformó con 16 de consumidores, el segmento 2 con 31 y el 3 con 13 consumidores. 


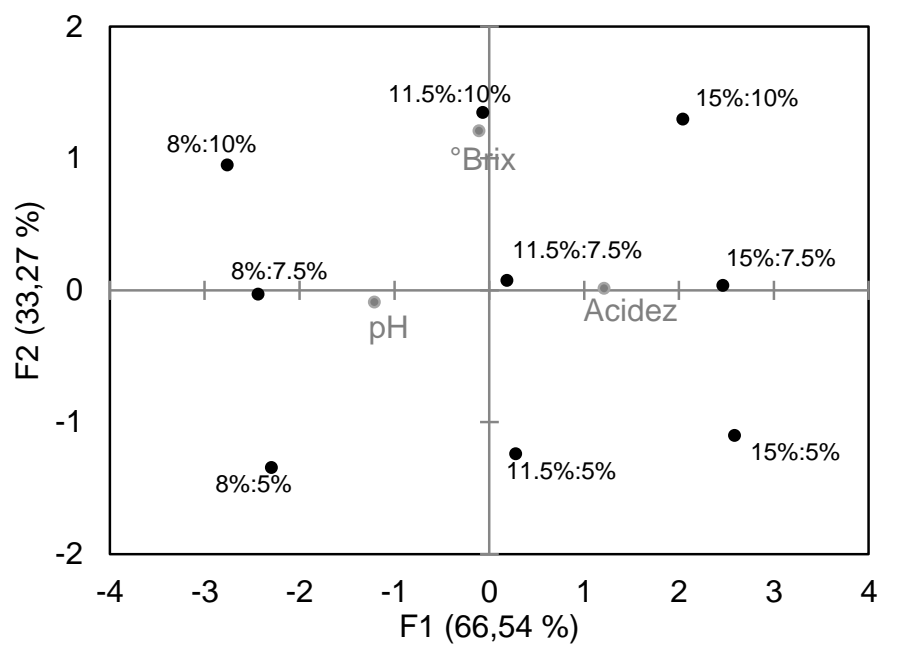

Fig. 1: Espacio físico-químico y formulaciones

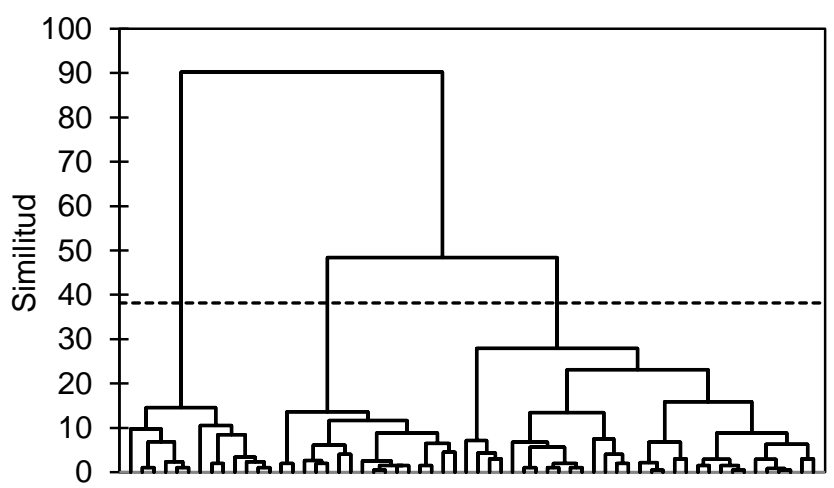

Fig. 2: Agrupaciones de consumidores

En el mapa externo de preferencia (Figura 3) modelo vectorial para la aceptación general, los consumidores del segmento 1 y 3 tuvieron un efecto significativo $\left(R^{2}=0.77\right.$ y $\left.R^{2}=0.88, p<0.05\right)$ ya que con dicho modelo se explicaron las preferencias de este grupo de consumidores ( 29 total) determinando su aceptación general en común por la bebida de formulación 11.5\%:10.0\% (pulpa:azúcar), de acuerdo a la característica físicoquímica de ${ }^{\circ}$ Brix que podemos asociar con la sensación dulce de la bebida. Los consumidores del segmento 2 tuvieron un efecto significativo $\left(\mathrm{R}^{2}=0.89, \mathrm{p}<0.05\right)$ del modelo vectorial exhibiendo la mayor aceptación por la formulación 15.0\%:10.0\% (pulpa:azúcar). Ramírez et al. (2010) obtuvieron resultados cuanto aplicaron los modelos vectoriales y circulares en investigación para explicar mejor la preferencia de los consumidores.

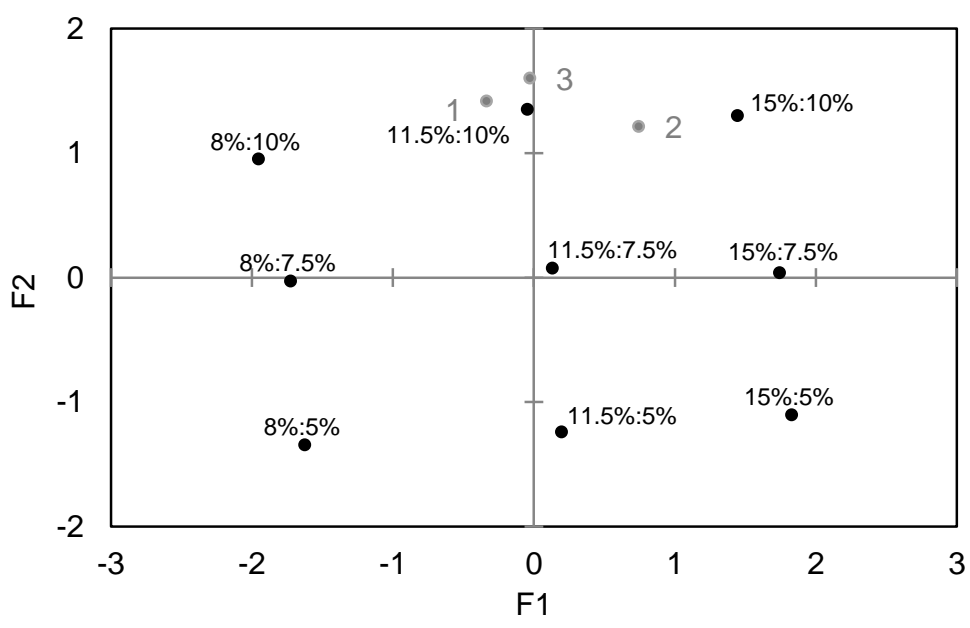

Fig. 3: Mapa externo de preferencia modelo vectorial 
En el mapa externo de preferencia (Figura 4) modelo vectorial, las medidas del grado de satisfacción del atributo sabor en los segmentos 1,2 y 3 tuvieron un efecto significativo $\left(R^{2}=0.85, R^{2}=0.88\right.$ y $\left.R^{2}=0.83, p<0.05\right)$; mientras los segmentos 2 y 3 tuvieron un efecto significativo $\left(R^{2}=0.70\right.$ y $\left.R^{2}=0.69, p<0.05\right)$ en el atributo olor, el segmento 1 no lo tuvo ( $p>0.05$ ) para describir el grado de satisfacción de los consumidores. Para el atributo color el segmento 1 fue significativo $\left(R^{2}=0.83, p<0.05\right)$ para el modelo vectorial, el segmento 2 para el modelo circular $\left(R^{2}=0.91, p<0.05\right)$ y no significativo para el segmento $3(p>0.05)$; además, de ser un grupo con pocos consumidores (4) se eliminó del estudio ya que el tamaño del grupo es pequeño y no representaba una porción significativa del total. Casos similares donde eliminaron grupos se presentaron en los estudios de Gambaro et al. (2007) en miel y Ramírez et al. (2010) en la formulación de salchichas.
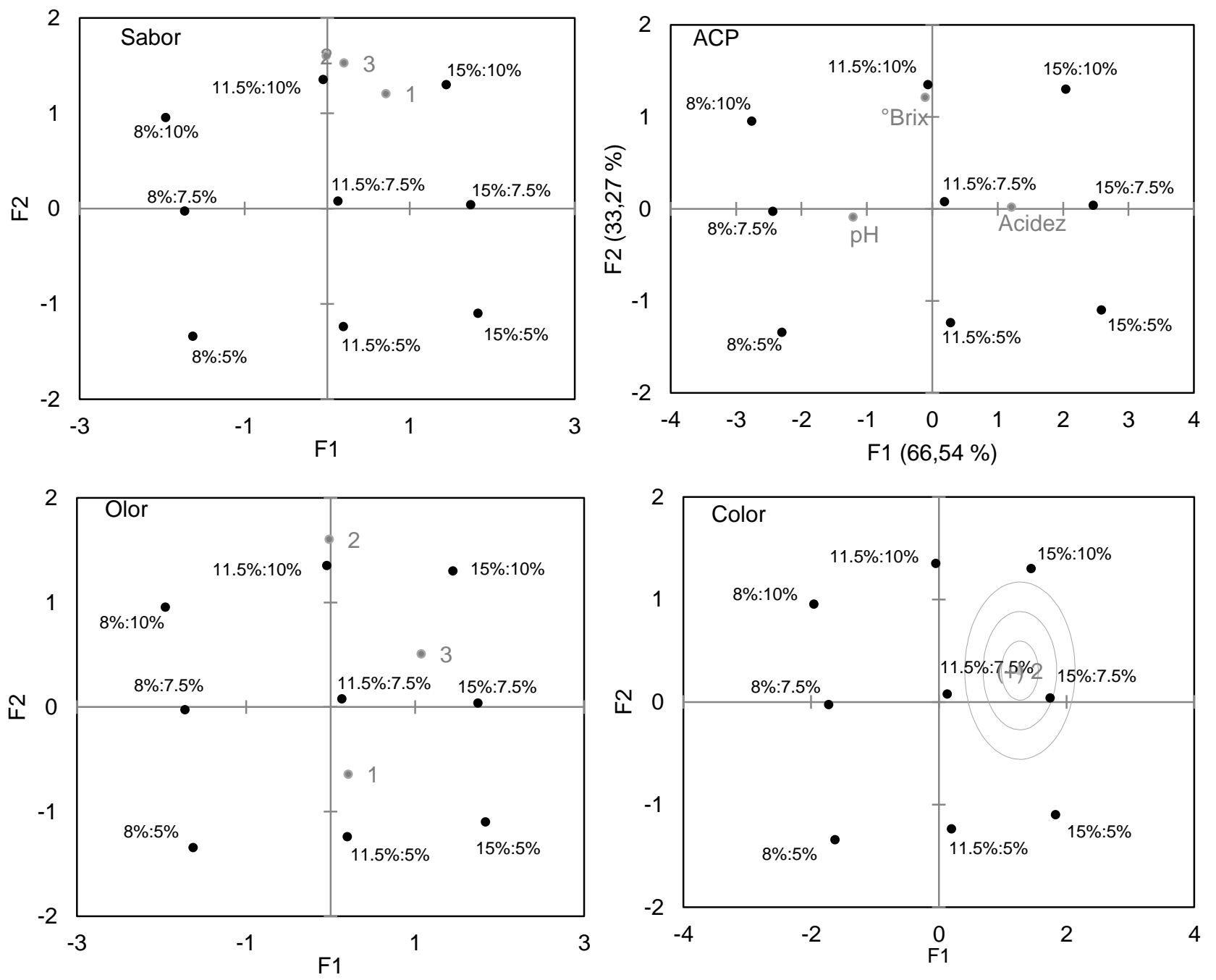

Fig. 4: Mapa de preferencia para atributos sabor, olor y color

Con respecto al atributo sabor la preferencia de los tres segmentos es homogénea respecto a las bebidas con 15\%:10\% y $11.5 \%: 10 \%$ asociadas con el parámetro ${ }^{\circ}$ Brix acorde al ACP. La preferencia de los consumidores con los atributos olor y color son más heterogéneos. Así, en el atributo olor el segmento 2 sigue la misma tendencia de preferencia del sabor por su ubicación en el cuadrante, mientras el segmento 3 se desplaza a las bebidas con un perfil más ácido acorde al ACP. En cuanto al atributo color se da un caso especial de la preferencia de los consumidores mostrando un punto ideal, según Resano et al. (2010) esto representa un punto máximo global en términos de preferencia. Padiyan et al. (2011) elaboraron bebida de lactosuero saborizado con pulpa de mango, donde la preferencia usando una escala hedónica de 9 puntos, donde la mayores notas superiores a 8 corresponden a la formulación con mayor pulpa de mango (6\%), Shukla et al. (2013) optimizaron la proporción jugo de piña y lactosuero obteniendo que la relación 35piña:65jugo fue la que mejor aceptación general $(8,93)$ por consumidores usando una escala hedónica de 9 puntos, correspondiendo a la mayor concentración de jugo, Mazhuvanchery y Kulkarni (2013) elaboraron un tea donde la mezcla $25 \%$ lactosuero y $8 \%$ de azúcar fue la más aceptable en términos de los atributos sensoriales (color, cuerpo, sabor, aceptación general) superior a 8 a partir de una escala de 9 puntos. 
En general, para cada uno de los atributos existe una preferencia (Tabla 3) en la cual las de mayor preferencia en común para los diferentes segmentos son las formulaciones $15 \%: 7.5 \%, 15 \%: 10 \%$ y $11 \%: 10 \%$, estas bebidas tienen en la formulación concentraciones de azúcar alta y se asocian con el sabor dulce que gusta al consumidor.

Tabla 3: Segmentos clasificados por orden ascendente de preferencia de formulaciones. Relación Pulpa:Azúcar en \%

\begin{tabular}{|l|l|l|l|l|l|l|l|}
\hline \multicolumn{3}{|c|}{ Sabor $^{\star}$} & \multicolumn{1}{c|}{ Olor $^{*}$} & \multicolumn{2}{c|}{ Color $^{*}$} \\
\hline \multicolumn{1}{|c|}{1} & \multicolumn{1}{|c|}{2} & \multicolumn{1}{c|}{3} & \multicolumn{1}{c|}{1} & \multicolumn{1}{c|}{2} & \multicolumn{1}{c|}{3} & \multicolumn{1}{c|}{1} & \multicolumn{1}{c|}{2} \\
\hline $8: 5$ & $8: 5$ & $8: 5$ & $8: 10$ & $8: 5$ & $8: 5$ & $8: 5$ & $8: 5$ \\
\hline $11.5: 5$ & $11.5: 5$ & $11.5: 5$ & $11.5: 10$ & $11.5: 5$ & $8: 7.5$ & $8: 7.5$ & $8: 10$ \\
\hline $8: 7.5$ & $15: 5$ & $15: 5$ & $15: 10$ & $15: 5$ & $8: 10$ & $8: 10$ & $8: 7.5$ \\
\hline $8: 10$ & $8: 7.5$ & $8: 7.5$ & $8: 7.5$ & $8: 7.5$ & $11.5: 5$ & $11.5: 5$ & $11.5: 5$ \\
\hline $15: 5$ & $15: 7.5$ & $11.5: 7.5$ & $11.5: 7.5$ & $15: 7.5$ & $11.5: 7.5$ & $11.5: 7.5$ & $11.5: 10$ \\
\hline $11.5: 7.5$ & $11.5: 7.5$ & $15: 7.5$ & $15: 7.5$ & $11.5: 7.5$ & $11.5: 10$ & $11.5: 10$ & $15: 5$ \\
\hline $15: 7.5$ & $8: 10$ & $8: 10$ & $8: 5$ & $8: 10$ & $15: 5$ & $15: 5$ & $11.5: 7.5$ \\
\hline $11.5: 10$ & $15: 10$ & $11.5: 10$ & $11.5: 5$ & $15: 10$ & $15: 7.5$ & $15: 7.5$ & $15: 10$ \\
\hline $15: 10$ & $11.5: 10$ & $15: 10$ & $15: 5$ & $11.5: 10$ & $15: 10$ & $15: 10$ & $15: 7.5$ \\
\hline
\end{tabular}

\section{CONCLUSIONES}

Las propiedades fisicoquímicas varían en las diferentes formulaciones de acuerdo a los porcentajes de pulpa y azúcar, así que entre mayor sea la cantidad de pulpa menor será el pH y por ende la acidez será mayor; los valores de $\mathrm{pH}$ y acidez reportados para las diferentes formulaciones están dentro de las especificaciones señaladas en la Resolución 3929 de 2013 para refrescos de fruta.

La relación entre evaluaciones sensoriales por consumidores y los datos instrumentales dieron información de las diferencias en las preferencias de los consumidores haciendo uso de la Clasificación Ascendente Jerárquica y el Mapa de Preferencia para discriminar grupos que prefieren la bebida más dulce, más acida 0 simplemente identificarse con un color óptimo en los cuadrantes del mapa.

Las formulaciones más aceptadas por los consumidores fueron la relación pulpa: azúcar de 15\%:7.5\%, 15\%:10\% y $11.5 \%: 10 \%$ respectivamente, lo que se relaciona con un sabor más dulce e intenso en términos de acidez, debido a que tienen unas concentraciones mayores de azúcar y de pulpa.

\section{REFERENCIAS}

Acevedo, D., J. Jaimes y C. Espitia, Efecto de la adición de lactosuero al queso costeño amasado, doi: 10.4067/S0718-07642015000200003, Información Tecnológica, 26(2), 11-16 (2015)

Álvarez, C., Caracterización fisicoquímica de los diferentes tipos lactosueros producidos en la Cooperativa Colanta LTDA, Tesis de licenciatura, Corporación Universitaria Lasallista, Colombia (2013)

A.O.A.C.: Association of Official Analytical Chemist, Official Methods of Analysis, Section 954.10. 13th. Ed. Washington D.C., USA (1993)

Cerquera, N., A. Coronado y J. Camacho, Determinación de variables de secado en lámina para la deshidratación de pulpa de maracuyá, Revista Colombiana de Ciencias Hortícolas, 6(2), 172-182 (2012)

Chóez, J., Elaboración de una bebida hidratante a base de lactosuero y enriquecida con vitaminas. Tesis Ingeniero de Alimentos, Escuela Superior Politécnica del Litoral, Ecuador (2010)

Conti, J. y otros tres autores, Perfil proteico y peptídico de una base fluida para bebidas funcionales obtenida por fermentación de lactosuero, doi: 10.4067/S0718-07642014000300007, Información Tecnológica, 25(3), 43-52 (2014) 
Coto, W. y F. Flores, Propuesta de Preformulación de una Bebida a base de Lactosuero y Frutas Naturales (Fresa y Maracuyá), Tesis Licenciatura en Química y Farmacia, Universidad de El Salvador, El Salvador (2011)

Gámbaro, A. y otros tres autores, Preferente Mapping of Color of Uruguayan Honeys, Journal Sensory Studies, 22, 507-519 (2007)

García, C., G. Arrázola y M. Villalba, Producción de Ácido Láctico de Lactosuero Suplementado utilizando Lactobacillus casei, Biotecnología en el Sector Agropecuario y Agroindustrial, 11(1), 136 - 143 (2013)

Hernández, A y A. Matos, Importancia del lactosuero en la industria de alimentos como bebida isotónica, Memorias I. Congreso Nacional de Investigación, Iglesia Adventista del Séptimo Día, Lima (2011)

ICONTEC NTC 404: Instituto Colombiano de Normas Técnicas y Certificación, Frutas Procesadas. Jugos y Pulpas de Frutas, Bogota, Colombia (1998)

Krasaekoopt, W. y T. Lokuliyanage, Effect of Hydrocolloids on Sensory Properties of the Fermented Whey Beverage from Different Types of Milk, AU J.T., 14(4), 253-258 (2011)

Lagua, H., Elaboración de una bebida nutritiva a partir de la pulpa de maracuyá (Pasiflora incarnata), y suero láctico, en la planta procesadora de frutas y hortalizas de la Universidad Estatal de Bolívar, Tesis Ingeniero Agroindustrial, Universidad Estatal de Bolívar, Ecuador (2011)

Londoño, M. y otros tres autores, Bebida fermentada de suero de queso fresco inoculada con Lactobacillus casei, Revista Facultad Nacional de Agronomía, 61(1), 4409-4421 (2008)

Mazhuvanchery, J. y S. Kulkarni, Development of Tea Whitener Using Concentrated Whey and Milk Solids, http://dx.doi.org/10.4236/fns.2013.42029, Food and Nutrition Sciences, 4, 215-221 (2013)

Pandiyan, C., R. Annal Villi y V. Chandirasekaran, Development of Mango Flavoured Sweetened Whey Drink, JIVA, 9(3), 35-37 (2011)

Pilaquinga, P., Desarrollo de una Bebida Refrescante con Frutas a Base de Lactosuero, Tesis Ingeniero de Alimentos, Universidad Tecnológica Equinoccial, Quito (2012)

Ramírez, E. y otros siete autores, Mapa externo de preferencias con datos sensoriales e instrumentales para la evaluación de salchichas de Euthynnus lineatus, Temas de Ciencia y Tecnología, 14(42), 19 - 28 (2010)

Resano, H. y otros cuatro autores, Sensory attributes that drive consumer acceptability of dry-cured ham and convergence with trained sensory data, Meat Sci., 84, 344-351 (2010)

Resolución 7992, Reglamento Técnico sobre la elaboración, conservación y comercialización de Jugos. Concentrados, Néctares, Pulpas, Pulpas Azucaradas y Refrescos de Fruta, 1-21, Bogotá, Colombia (1991)

Resolución 3929, Reglamento técnico sobre los requisitos sanitarios que deben cumplir las frutas y bebidas con adición de jugo (zumo) o pulpa de frutas, 1-29, Bogotá, Colombia (2013)

Sameen, A. y otros tres autores, Effect of Stabilizers on the Quality of Carbonated Flavoured Whey Drink, Afr. J. Agric. Res., 8(5), 445-448 (2013)

Shukla, M., Y. Kumar Jha, y S. Admassu, Development of Probiotic Beverage from Whey and Pineapple Juice, doi:10.4172/2157-7110.1000206, J Food Process Technol., 4(2), 1-4 (2013)

Singh, A. y K. Singh, Utilization of Whey for the Production of Instant Energy Beverage by Using Response Surface Methodology, Advance journal of Food Science and Technology, 4(2), 103-111(2012) 